\title{
Robotic thoracic surgery in inflammatory and infective diseases
}

\author{
Ali Zamir Khan ${ }^{1}$, Sangeeta Khanna ${ }^{2}$, Narendra Agarwal ${ }^{1}$, Kamran Ali $^{1}$ \\ ${ }^{1}$ Department of Minimally Invasive Thoracic Surgery, ${ }^{2}$ Department of Anesthesia, Medanta, The Medicity, Gurgaon, India \\ Correspondence to: Dr. Ali Zamir Khan. Department of Minimally Invasive Thoracic Surgery, Medanta, The Medicity, Sector 38, Gurgaon 122001, \\ India. Email: azamirk@gmail.com.
}

\begin{abstract}
Tuberculosis and inflammatory conditions are endemic in India and South-East Asia. They cause intense inflammatory reactions and adhesions, thus making surgical resection difficult. In 2009, we installed an intuitive da Vinci HDSi robot to perform our surgery as a part of a robotic thoracic surgery unit. We reviewed our practice to report the trials and tribulations of starting a robotic thoracic surgery program in an inflammatory and infective disease endemic third-world country. With the success of the multispecialty robotic surgery program, we were able to purchase a second robot with an operating console and a training console. The robot is an additional tool in the armamentarium of the thoracic surgeon. It provides good vision in inflammatory conditions, facilitates dissection of dense adhesions with minimal blood loss, and the ability of the robotic endowrist allows maximum and safe manipulation at the thoracic outlet. Sleeve resection and sleeve lobectomy are technically possible, due to ease of suturing with the robotic platform. Complex resections for tuberculosis, aspergilloma, inflammatory tumours and post-infective bronchiectasis are safer using the robotic thoracic platform. This is our operation of choice in complex thoracic surgery cases.
\end{abstract}

Keywords: Robotic thoracic surgery; inflammatory diseases; tuberculosis; chest wall adhesions

Submitted Jan 27, 2019. Accepted for publication Feb 20, 2019.

doi: 10.21037/acs.2019.02.05

View this article at: http://dx.doi.org/10.21037/acs.2019.02.05

\section{Introduction}

Inflammatory diseases are prevalent in in South-East Asia. They cause dense adhesions in the chest and are technically challenging in the context of video assisted (VATS) and robotic thoracic surgery (RTS). In India, there were many challenges to setting up a robotic thoracic surgery program. These include:

(I) Clinical diagnosis of multidrug resistant (MDR) tuberculosis, extensively drug resistant (XDR) tuberculosis, aspergilloma and associated bronchiectasis;

(II) Destroyed lungs and adhesions;

(III) Poor nutritional status of patients undergoing complex thoracic procedures;

(IV) Cost of procedures including installation, running and disposables costs, such as robotic drapes and staplers;

(V) Paucity of expertise and training programs;

(VI) The lack of availability of trained thoracic surgeons.
General surgeons fill the gap by practicing thoracic surgery.

Trained surgeons with expertise in minimally invasive techniques are returning back to their home country from the US and Europe, bringing minimally invasive surgical expertise. This facilitates the setting up of robotic thoracic surgery programs, despite the high incidence of inflammatory diseases.

\section{Robotics in tuberculosis and its sequelae}

Robotic thoracic surgery has been performed at our hospital for the following sequelae:

(I) Robotic lobectomy for aspergilloma in a posttuberculous cavity;

(II) Robotic lobectomy/segmentectomy for posttuberculosis bronchiectasis;

(III) Robotic lobectomy for lung abscess;

(IV) Robotic resection of infected bronchogenic cysts;

(V) Robotic drainage of multi-loculated empyema and 

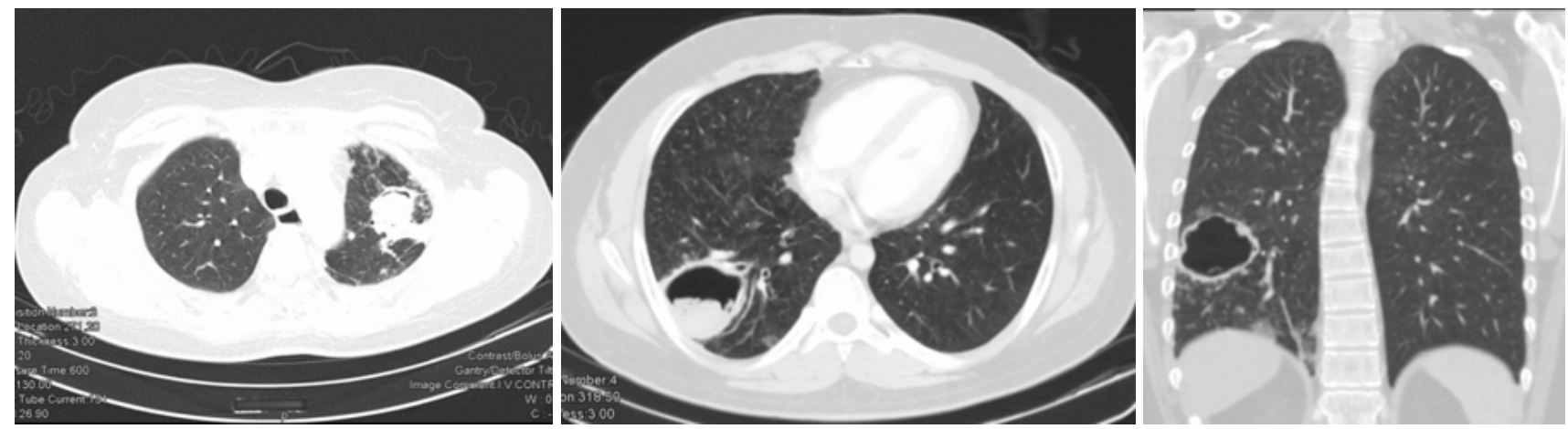

Figure $1 \mathrm{CT}$ scan showing a post-tuberculosis aspergilloma.

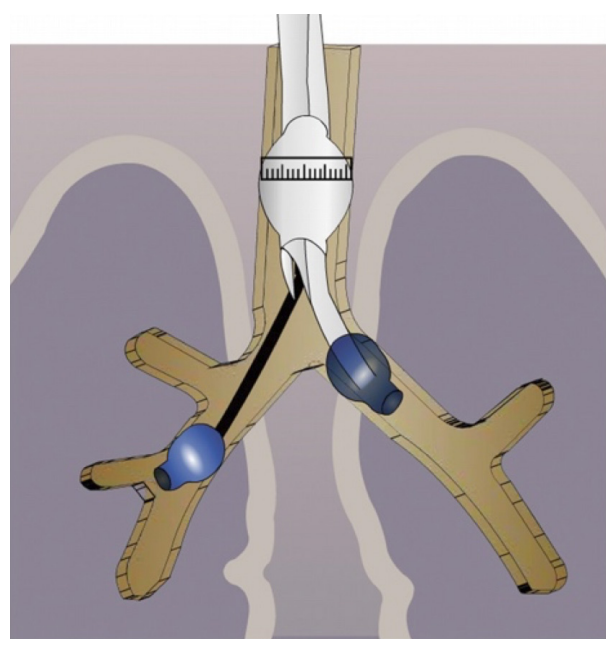

Figure 2 Anesthetic tricks to prevent endobronchial spillage of Aspergilloma (4). Image courtesy: Dr. Preety Mittal Roy (4).

decortication;

(VI) Robotic wedge resection of residual tuberculous cavities predisposing to hemoptysis;

(VII) Robotic lung volume reduction surgery for emphysematous post-tuberculosis lung;

(VIII) Robotic resection of post-tuberculosis giant bullae.

\section{Robotic resection of post-tuberculosis aspergilloma}

Pulmonary aspergilloma (Figure 1) is usually difficult to treat with antifungals only. Surgical resection of aspergilloma is likely to result in a complete cure. It is technically challenging, owing to dense adhesions, frozen hilar structures and intraoperative bleeding. The mortality following surgery is also significant. There has been a constant evolution in the search of the most appropriate surgical approach for pulmonary aspergilloma. Minimally invasive thoracic surgery for such cases has been reported in the literature with many centers around the world now adopting video-assisted thoracic surgery (VATS) pulmonary resections in selected cases $(1,2)$. However there are few reported cases of robotic resection for pulmonary aspergilloma (3).

New anesthetic strategies to prevent endobronchial \& systemic spillage in aspergilloma

Aspergilloma may sometimes have endobronchial communications. While the patient is awake, the cough reflex prevents spillage to other lobes or opposite lungs. As soon as the patient is induced, there is a risk of endobronchial spillage into the opposite lung or other lobes. We experienced this in a ten-year-old child. Due to cross contamination, it was difficult to ventilate the patient during surgery. The patient subsequently developed systemic fungal septicemia.

To overcome these problems, we developed a strategy of placing a double lumen tube with the patient in the lateral position with the affected side down. We then turn the patient on his back and place a Fogarty catheter into the affected side to isolate the non-affected lobes of the lung (Figure 2) (4). This protects the other lobes on the same side as well as the contralateral lung. During surgery, it is important to ask the anesthetist to remove the Fogarty catheter before stapling the bronchus.

\section{Operative technique (Video 1)}

Following the incisions and port placement (Figure 3), 


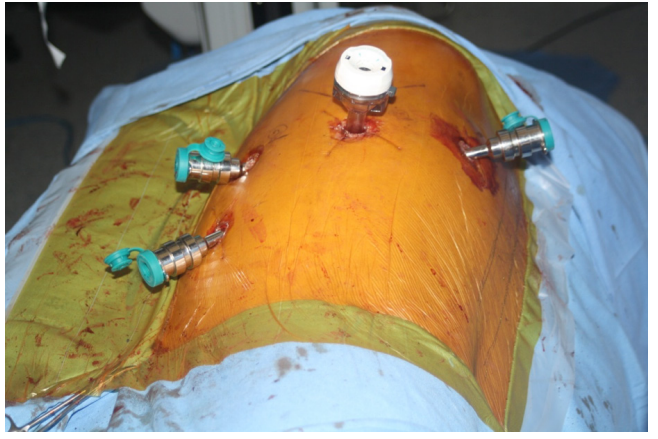

Figure 3 Port placement for robotic resection of pulmonary aspergilloma.

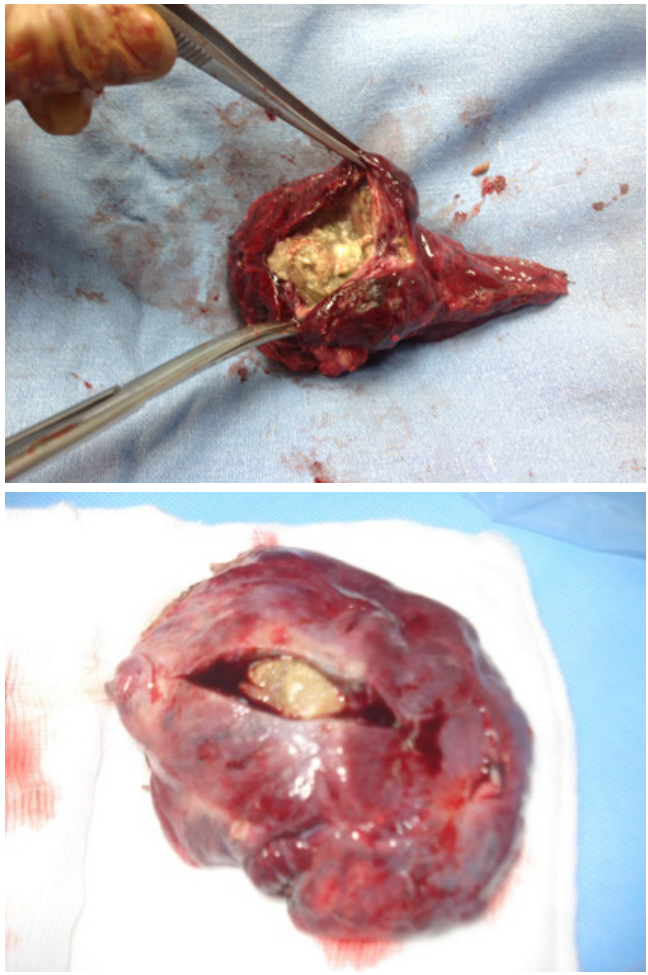

Figure 4 Cut section showing yellow aspergilloma.

a four arm da Vinci Robot is docked to the patient and adhesiolysis performed. Dense adhesions should be dissected only enough to facilitate the docking of the robot. Adhesions at the apex should not be taken down initially. They allow good retraction of the lobe and facilitate work at the hilum. The adhesions are highly vascular due to neovascularization. Dissecting them early on during the operation leads to bleeding and obscured vision.

It is mandatory that the lobe is not manipulated
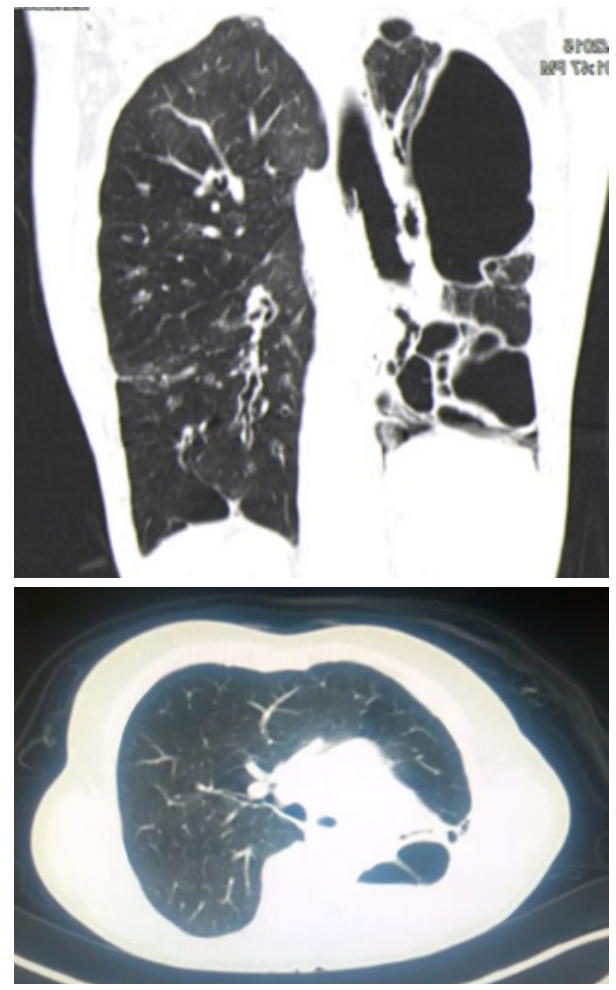

Figure 5 Destroyed Tuberculosis Left Lung.

during dissection as this may cause systemic spillage of aspergilloma. The pulmonary vein is first dissected and divided using an endoscopic stapler. This is followed by the division of lobar bronchus. It is imperative to staple the vein and bronchus as soon as possible to prevent endobronchial and systemic spillage of the aspergilloma. The anesthetist is requested to withdraw the Fogarty catheter to prevent accidental stapling of the catheter to the bronchus. All arterial branches to the affected lobe are carefully dissected \& divided later. Dense adhesions of the apex to the upper lobe are then divided. The robot is then undocked. The utility incision is extended to facilitate the delivery of the specimen (Figure 4) from the chest in an endobag (locally made, refashioned urinary bag).

\section{Robotic pneumonectomy for destroyed lung}

Tuberculosis patients often present with hemoptysis with a background of destroyed lung tissue (Figure 5). In our practice, these patients are offered a robotic lobectomy or pneumonectomy (Figure 6), depending on the extent of the disease. The adhesions in tuberculosis are usually more at the periphery and at the diaphragm. Our experience 


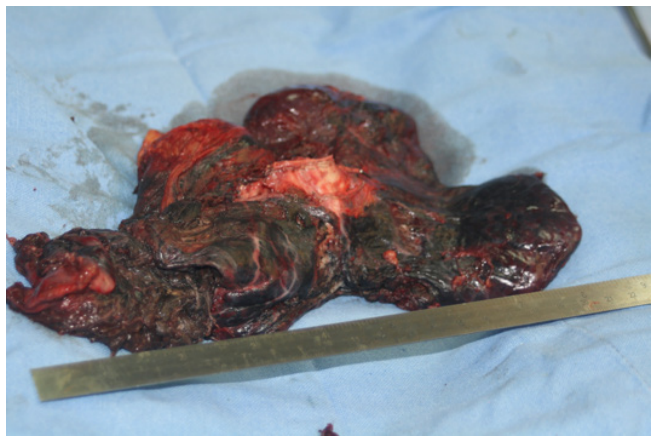

Figure 6 Robotic Pneumonectomy for tuberculosis destroyed left lung.
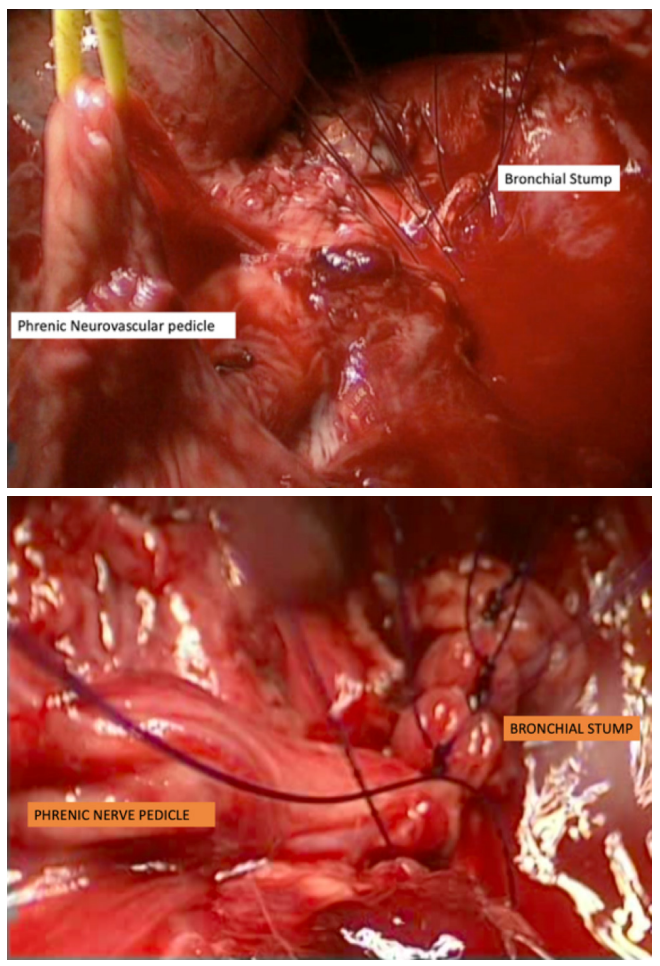

Figure 7 Phrenic neurovascular bundle for reinforcing a pneumonectomy stump.

suggests that the hilum is surprisingly spared. In two cases, the hilum was frozen and intra pericardial stapling of the pulmonary vasculature was performed.

This series also includes a robotic pneumonectomy performed in a case of left hilar squamous cell adenocarcinoma of lung, down-staged with radical radiotherapy. The patient also had a history of a coronary artery bypass graft and a patent, functioning left internal

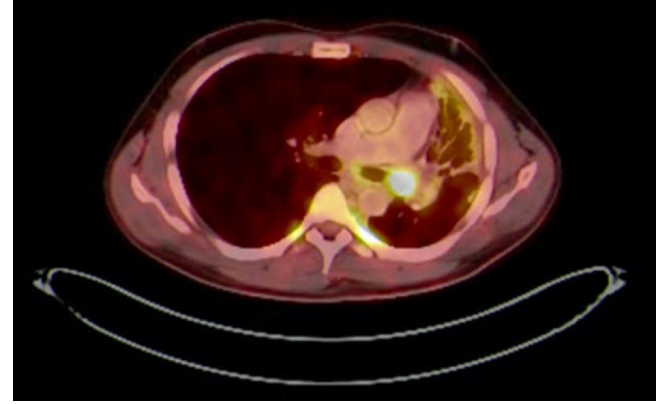

Figure 8 PET CT showing presence of left main bronchus inflammatory pseudo tumour.

mammary artery graft to the left anterior descending artery of the heart. The extent of adhesions and subsequent dissection was similar to performing a surgery on inflammatory conditions.

\section{Use of pedicle to reinforce bronchial stump}

Previous radiotherapy, multi-drug resistant tuberculosis (MDR TB) or persistent endobronchial culture positive tuberculosis despite adequate treatment (Mycobacterium other than tuberculosis: MOTT) are indications for harvesting of an intercostal muscle pedicle or pericardium to cover the bronchial stump. In two cases of robotic pneumonectomy and seven cases of video assisted pneumonectomy, we used a phrenic neurovascular pedicle harvest to reinforce the bronchial stump (Figure 7). This give the required reinforcement as well as allowing for the diaphragm to rise up to reduce the post-pneumonectomy space.

\section{Robotic sleeve lobectomy for inflammatory pseudotumour}

A 26-year-old patient presented to us from Bangladesh with a history of recurrent chest infections.

PET CT scan showed the presence of an endobronchial tumour in the left main bronchus (Figure 8). There was some aeration of left lower lobe with a collapsed, consolidated upper lobe. An endobronchial biopsy was performed. Histopathology suggested a preliminary diagnosis of endobronchial inflammatory pseudotumor. Acid fast bacilli were negative on microscopy and culture. He was scheduled for robotic sleeve resection of lung: a sleeve lobectomy or a sleeve resection to save the left 

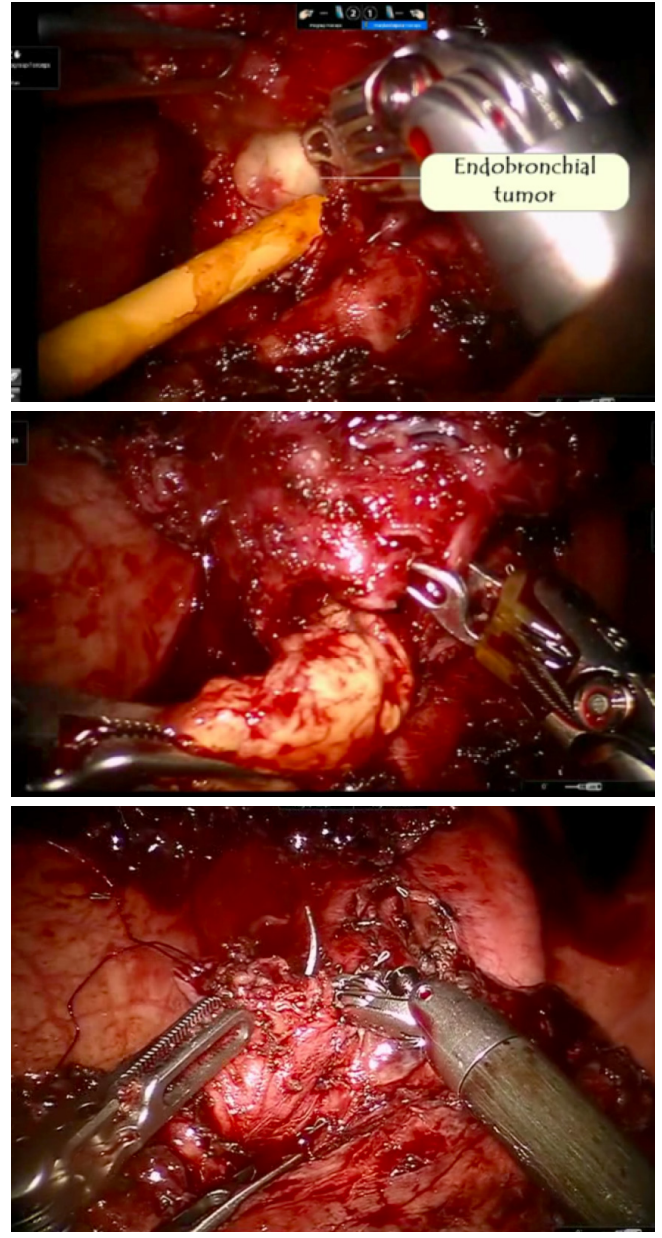

Figure 9 Intraoperative robotic bronchotomy \& removal of tumour.

lower lobe.

\section{Intraoperative strategy (Video 2)}

A preoperative rigid and flexible bronchoscopy was performed. The growth seemed to arise from the left upper lobe and encroached into the left main bronchus. Bronchoscopy suggested that the left lower lobe bronchus was blocked due to prolapsing tumour rather than infiltration.

An Intuitive da Vinci HDSi robotic platform was used to perform the surgery.

The surgery was started with VATS to enable some dissection of adhesions and create the space to dock the robot. With robotics, the adhesions were dissected under direct vision. The pulmonary artery and vein to upper

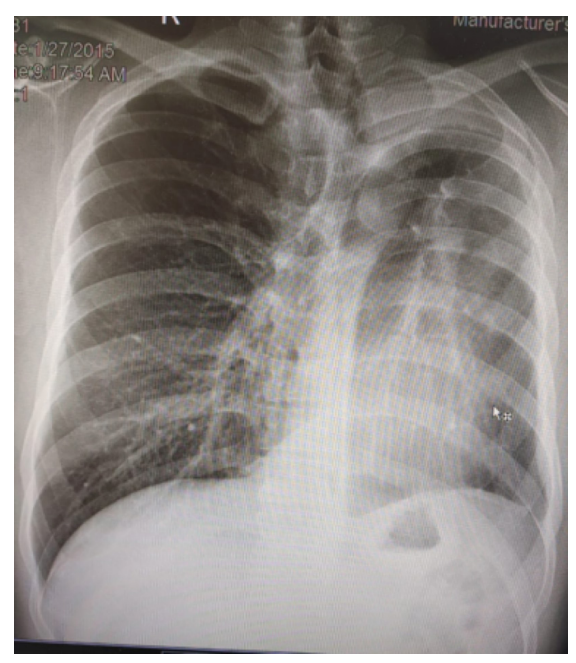

Figure 10 Follow up x-ray at 2 years post robotic sleeve lobectomy.

lobe was dissected and stapled. The bronchus to the upper lobe was isolated. A bronchotomy was made using a robotic scissor. The tumor was delivered out from the bronchotomy. An intraoperative flexible bronchoscopy was performed to ensure complete removal of all tumor from lower lobe. Endobronchial suctioning and toilet of the lower lobe was performed. The upper lobe was resected and delivered out in a bag. The margins of the upper lobe bronchus were sutured with 3-0 PDS suture (Figure 9).

Use of the robotic platform to suture the bronchus is technically intuitive. It simulates suturing like open surgery. Intraoperative lung expansion and under water test was performed to ensure no air leak.

\section{Follow up}

This patient has been followed up at two years. Clinically, he is doing well with no symptoms of shortness of breath or recurrent infections. Chest X-ray confirms good lung expansion (Figure 10). Bronchoscopy is normal.

\section{Robotic decortication in multi loculated pleural effusion}

We have performed three cases of robotic decortication to explore the feasibility of this surgery using the robotic platform. It is technically feasible and safe.

Our preferred method is still VATS (635 patients) due to:

(I) Cost of the robotic platform. We reserve the robot for more complex cases; 

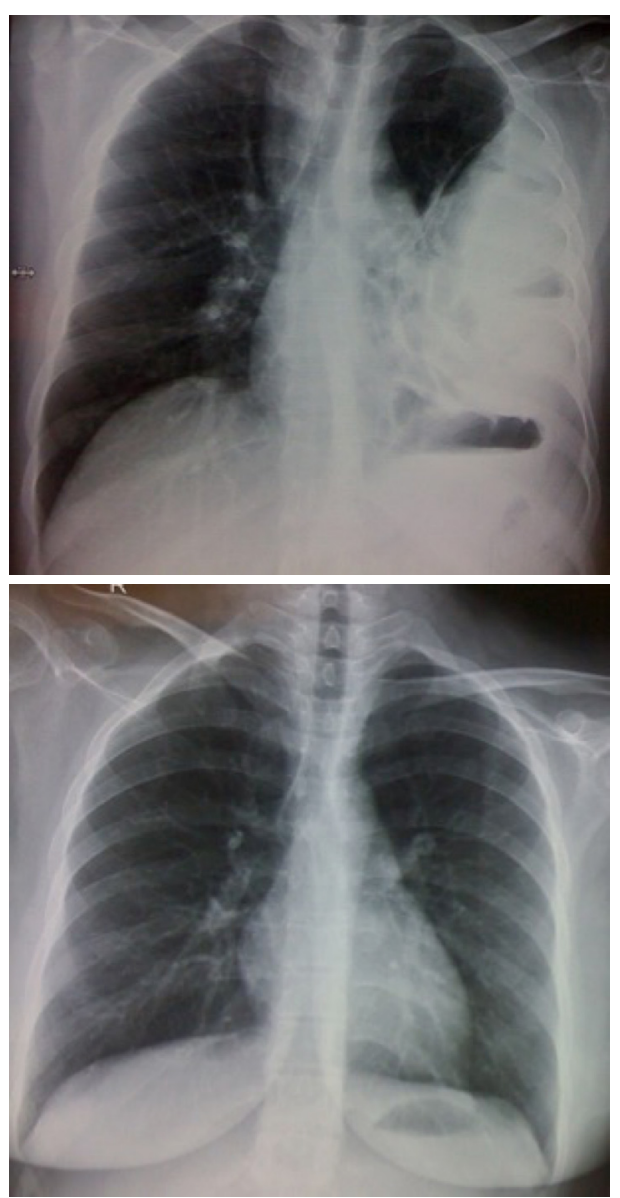

Figure 11 Preoperative Chest X-ray and 3 months postoperative chest X-ray following robotic decortication in a patient of tuberculous empyema.

(II) Loculations of empyema need different placement of ports in each case and hence, there may be difficulty in standardizing the robotic ports;

(III) Patient choice.

\section{Operative strategy}

Preoperative, detailed interpretation of chest CT scan is essential (Figure 11). Pus is identified by aspiration with a needle. Samples of the pus are collected for routine microbiology culture, sensitivity and AFB culture. Three ports are placed under VATS guidance. The robot is now docked. The lung is dissected off the chest wall. The hilum is freed all around. It is difficult and cumbersome to dissect the lung off the diaphragm due to dense tuberculous adhesions. The thick visceral cortex is incised under direct

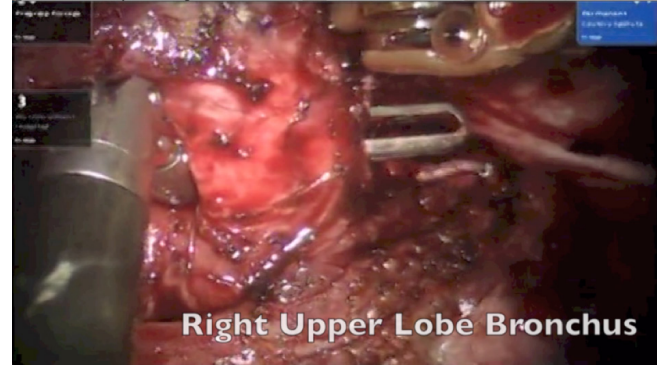

Figure 12 Robotic Lobectomy for tuberculous bronchiectasis.

vision up to the lung tissue. The cut end of the cortex is grasped and peeled off to free the trapped underlying lung. Gentle insufflation of the lung by the anesthetist facilitates the peeling of the cortex. The expansion of the lung is confirmed by double lung ventilation. The anesthetist has to apply positive pressure ventilation to re-expand the collapsed lung.

The entire pleural cavity is washed rigorously with a solution of one liter of saline with $100 \mathrm{~mL}$ of betadine and fifty milliliters of hydrogen peroxide. This procedure is repeated two to three times until complete hemostasis is achieved. We have used hydrogen peroxide in the pleural cavity in over a thousand cases. Two patients experienced an allergic reaction necessitating therapy.

\section{Robotic lobectomy/segmentectomy for post tuberculosis bronchiectasis}

These patients present with hemoptysis and recurrent chest infections following tuberculosis. CT scan confirmed lobar or segmental bronchiectasis. A robotic lobectomy/ segmentectomy was performed in these patients. This surgery is technically challenging due to dense adhesions between artery and bronchus (Figure 12). In three patients, it was not possible to dissect between the artery and bronchus. The artery and bronchus were stapled in a single stapler side by side rather than overlapping. This prevents a broncho-vascular fistula.

\section{Robotic resection of giant bullae/tuberculous cavity}

Tuberculous patients often develop giant bullae (Figure 13). We performed a robotic resection of giant bullae. Frequently these tuberculous cavity lesions are calcified. They require stapling with a black cartridge due 

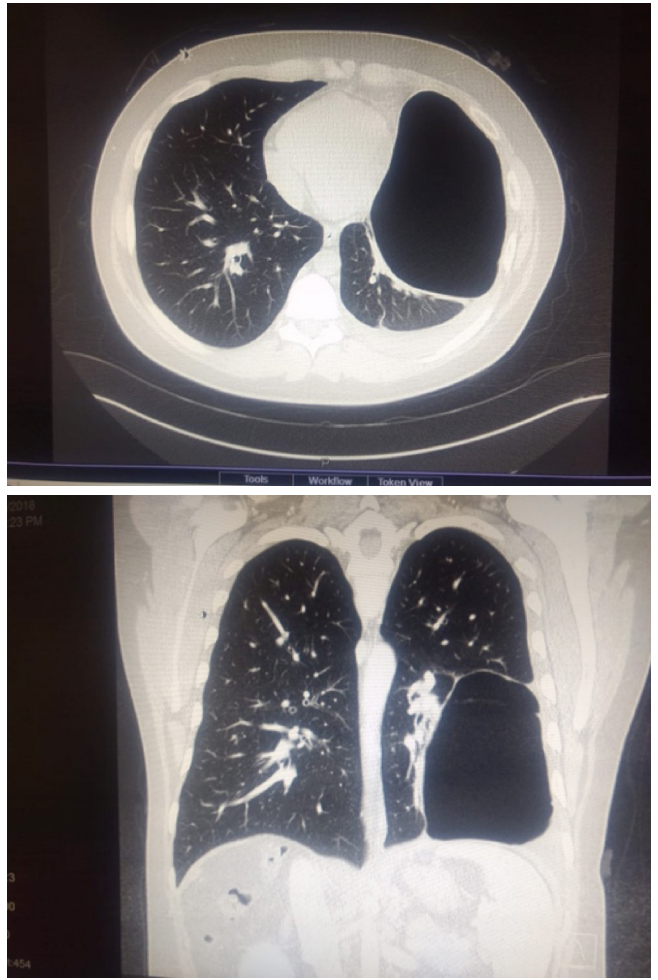

Figure 13 Post-Tuberculosis giant bulla.
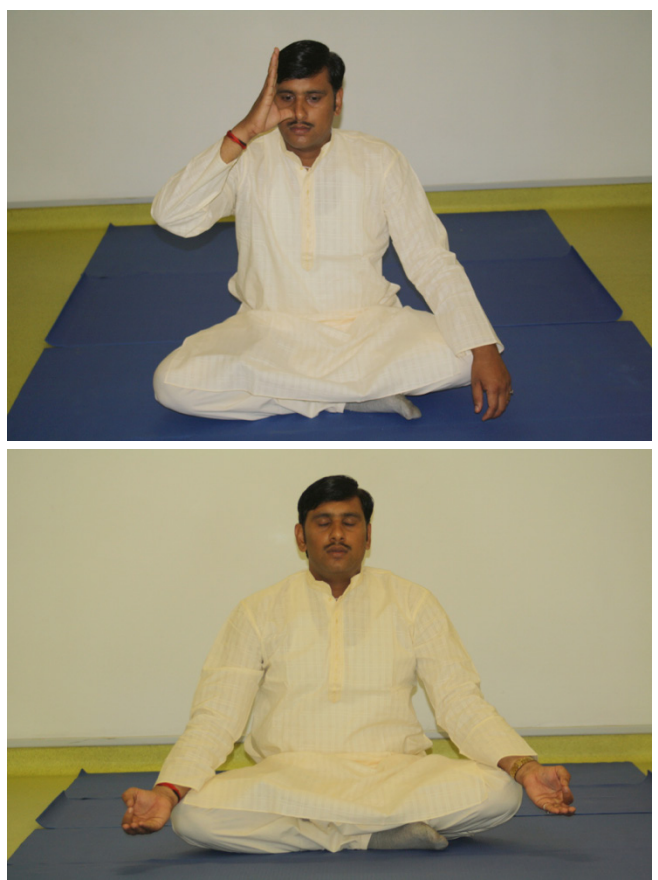

Figure 14 Use of Meditation, Yoga and Ayurveda in patients undergoing robotic surgery. to the thickness of the tissue.

\section{Robotic lung volume reduction surgery in inflammatory disease}

With high incidence of tuberculosis, smoking and pollution in India, emphysematous lung disease and segmental destroyed lungs are a common occurrence. Symptomatic patients with severe dyspnea and localized disease are considered for robotic lung volume reduction surgery. Preoperatively these patients are put through an intensive six-week course of physiotherapy and pulmonary rehabilitation. We usually operate one side at a time. A crescent along the lateral margin to the apex is stapled using staplers reinforced with pericardial buttress. Postoperative care includes aggressive physiotherapy, yoga, use of low volume low pressure suction, and early mobilization.

\section{Use of yoga and ayurveda (integrated medicine) for postoperative recovery}

The Integrative medicine/Ayurveda physician and Yoga therapist visited the patient within six hours of surgery in the postoperative room or the intensive care unit. The patients were encouraged to perform specific Yoga exercises (Figure 14) (Pranayam, Sookshmavyayam, Bhastrika, Anulom-Vilom and Nadeeshodhan) (5). This encouraged deep breathing and expectoration of phlegm. Yoga is administered with progressive intensity of breathing and physical mobilization, through the post-surgical admission, and continued daily and regularly until discharge and also at home. Patients were also taken outdoors to a rooftop, scientifically designed ayurvedic garden (Figure 15) growing medicinal plants used in Ayurveda.

\section{Integrative medicine for port site pain}

Port site pain was addressed with Ayurvedic therapies called "Urotarpan" (retaining warm medicated oil on specific area of chest for a specified period of time) (Figure 16) and "Patra Potali" (i.e., patra means medicated leaves and potali refers to a cloth bag where these special leaves are processed and bound-in (5). These potalis were warmed by immersing them in hot medicated oil, and thereafter used to give hotfomentation over the affected body part) (5) (Figure 16). A total of 854 patients (Complex VATS and Robotics) were put through the Yoga and Ayurvedic therapies. All patients 


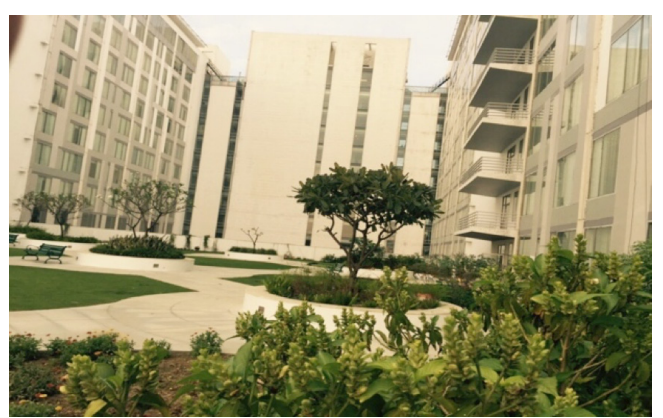

Figure 15 Ayurvedic garden for post-operative rehabilitation of robotic surgery patients.
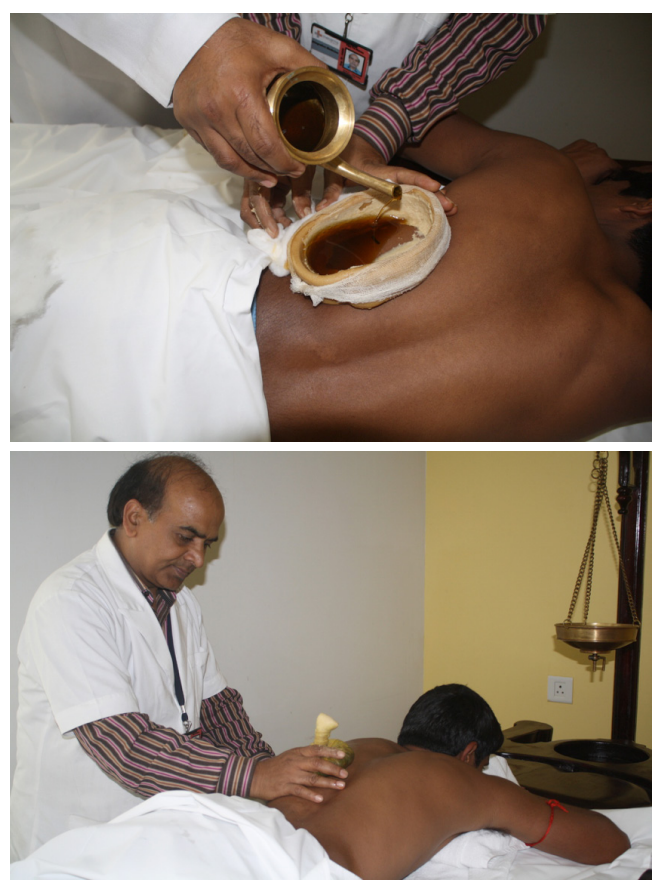

Figure 16 Use of Urotarpan and Patra Potali to treat post op pain.

showed complete resolution of symptoms.

\section{Morbidity and mortality}

One patient developed an intraoperative tension pneumothorax which was corrected by the release of $\mathrm{CO}_{2}$ insufflation. This was related to the abdominal default insufflator setting of $16 \mathrm{mmHg}$ and 16 liters of flow. Thoracic setting is $6 \mathrm{mmHg}$ and 6 liters of flow. This happened due to switch over from main supply to uninterrupted power source (UPS) during electricity failure.

One eighty-year-old patient in this series died due to secondary sepsis twenty days after surgery. Two patients required conversion to open thoracotomy due to a frozen hilum and bleeding. One patient required re-exploration by VATS for bleeding. One patient required re-exploration by VATS for sealing of air leak. All patients have done well and are asymptomatic at follow-up. Antituberculous medications have been started on all patients and are under follow up with pulmonologist. All patients have responded to their antituberculous regime.

\section{Cost reduction in robotic surgery in third world countries}

In South-East Asia, cost remains an important hindrance for robotic surgery. We address the cost issues as follows:

(I) We keep the robot busy by instituting multidepartment use. Our hospital has two dedicated robotic theatres which function from 8 am to at least $8 \mathrm{pm}$. Urology, gynecology, thoracic surgery, otolaryngology all contribute to keep the robot busy. This makes it cost effective in terms of maintenance costs;

(II) In thoracic surgery, we preferentially use 3 arms instead of 4 arms. The judicious use of instruments reduces the cost of the operation. Assistance and retraction is done by the surgeon at the table;

(III) Robotic staplers are expensive and use of standard non-robotic staplers reduces the cost;

(IV) Robotic suturing and tying of small vessels also reduces the number of staplers used;

(V) Use of a locally made bag out of a simple cut to size urinary bag reduces the cost of disposable prefabricated endoscopic bags.

\section{Discussion}

In patients with inflammatory diseases, surgical resection is generally considered technically very challenging due to the presence of dense adhesions. These patients are difficult to dissect and have the potential to bleed significantly. Bleeding can obscure the vision as well as add to the morbidity. Inflamed and indurated hilar structures due to extrabronchial fibrosis and post-chronic infection nodes make the surgeon's task even more difficult.

Traditionally, the surgical approach for aspergilloma is through a liberal thoracotomy. However, the last decade has seen a rise in the number of VATS pulmonary resections performed for aspergilloma $(1,2)$. A recent 
retrospective study compared VATS in the treatment of simple mycetoma and complex mycetoma. They concluded that VATS can be safely applied to simple aspergilloma and complex aspergilloma without infiltration of the hilum and allows for an early postoperative recovery $(2,3)$. Robotic surgery in inflammatory diseases (6) offered the following benefits:

(I) High-definition optics and 3D visualization of intrathoracic structures;

(II) The 360-degree movement and 7-degree freedom of the endowrist is helpful at the thoracic outlet to dissect dense adhesions without injury to subclavian vessels and brachial plexus;

(III) Adhesiolysis under vision thereby reducing blood loss;

(IV) Ability to dissect carefully under direct vision around major vascular structures in a frozen hilum;

(V) Ability to safely open the pericardium and perform an intra-pericardial stapling of pulmonary vasculature;

(VI) Ability to do a bronchotomy and deliver the tumor out so that a lobe could be spared;

(VII) The ability to suture the bronchus intuitively or perform a sleeve anastomosis.

Disadvantages of robotic systems are the learning curve and cost. It is mandatory to train all the staff to perform for emergency undocking of the robot. Multiple published articles have shown the efficacy and safety of robotic pulmonary resection including lobectomy, segmentectomy, and pneumonectomy (3). However. little data exists in the literature for robotic non-oncologic lung resections in inflammatory pathology. This is the first experience of use of robotic surgery in inflammatory and infective pathologies in a third world country. We hope that our experience will encourage other surgeons to perform robotic thoracic surgery for inflammatory diseases.

\section{Conclusions}

The robot is an additional tool in the armamentarium of the thoracic surgeon. It provides good vision in inflammatory conditions, facilitates dissection of dense adhesions with minimal blood loss and the robotic endowrist allows maximum and safe manipulation at the densely stuck thoracic outlet. Sleeve resection and sleeve lobectomy are technically possible, due to ease of suturing with the robotic platform. Complex resections for tuberculosis, aspergilloma, inflammatory tumours, and post-infective bronchiectasis are safe using the robotic thoracic platform. We can achieve good clinical outcomes and early discharge from hospital, making this our operation of choice in complex, inflammatory thoracic surgery patients.

\section{Acknowledgements}

None.

\section{Footnote}

Conflicts of Interest: The authors have no conflicts of interest to declare.

\section{References}

1. Ichinose J, Kohno T, Fujimori S. Video assisted thoracoscopic resection of Pulmonary aspergilloma. Interact Cardiovasc Thorac Surg 2010;10:927-30.

2. Chen QK, Chen C, Chen XF, et al. Video assisted thoracoscopic resection for pulmonary aspergilloma: a safe and effective procedure. Ann Thorac Surg 2014;97:218-23.

3. Khan AZ, Ali K, Khandelwal S, et al. Robotic assisted thoracoscopic right upper lobectomy for post tuberculosis aspergilloma. J Vis Surg 2016;2:51.

4. Roy PM, Khanna S, Mehta Y, et al. Aspergilloma of the Lung: Strategy to Prevent Endobronchial Spillage. Innovations (Phila) 2016;11:373-5.

5. Khan AZ, Pillai GG. From 200 BC to 2015 AD: an integration of robotic surgery and Ayurveda/Yoga. J Thorac Dis 2016;8:S84-92.

6. Yablonskii P, Kudriashov G, Vasilev I, et al. Robotassisted surgery in complex treatment of the pulmonary tuberculosis. J Vis Surg 2017;3:18.
Cite this article as: Khan AZ, Khanna S, Agarwal N, Ali K. Robotic thoracic surgery in inflammatory and infective diseases. Ann Cardiothorac Surg 2019;8(2):241-249. doi: 10.21037/ acs.2019.02.05 